\title{
Segmentation of the Heart Ventricle and Atrium in Handheld Ultrasound Images
}

\author{
Jun-Young Park ${ }^{1}$, Woo-Hyuk Choi ${ }^{1}$, Sung-Yun Park ${ }^{2}$ \\ ${ }^{1}$ Biomedical engineering Lab., Dongguk university \\ Dongguk-ro 32 Ilsandong-gu, Goyang, Republic of Korea \\ pipen0125@gmail.com; wowoohyuk@gmail.com \\ ${ }^{2}$ College Korean medicine, Dongguk university \\ Dongguk-ro 32 Ilsandong-gu, Goyang, Republic of Korea \\ bmepark@dongguk.ac.kr
}

\section{Extended Abstract}

Ultrasound is widely used to diagnose a heart disease due to non-invasive method and real time [1-4]. The handheld ultrasound is rapidly increasing on small or private hospital because of mobility, compact and cheaper than premium model.[5] This purpose of this study is to develop an automate segmentation algorithm that uses a measure a volume of heart ventricle and atrium.

The commercial device that is developed our own research centre is used to take a heart ventricle and atrium image. 6 males who have any cardiovascular disorders for 2 years were enrolled on this study. The algorithms of ultrasound image were developed based on MATLAB. The commercial device (ACUSON X300, Siemens, Germany) was used to check a performance of developed algorithm. The image analysis algorithm consists of 3 steps which are a pre-processing, separate region and segmentation. The pre-processing step includes a data size reduction and image enhancement. The separate region step includes a finding contours and convex hell, composite process between binary image and convex hull image, and extract ROI section. The segmentation step includes a calculating adaptive radius and a measuring a volume on each ventricle and atrium. The value (mean \pm SD) of EDV, ESV, stroke volume and EF on one subject were $2.20 \pm 0.05,0.78 \pm 0.1,1.42$ \pm 0.12 , and $64.65 \pm 2.14 \%$, respectively. The accuracy (mean \pm SD) of EDV, ESV, stroke volume and EF were $88.23 \pm$ $1.71 \%, 88.36 \pm 2.14 \%, 89.12 \pm 0.27 \%$, and $89.54 \pm 0.48 \%$. Further study, we will optimize the algorithm to increase the accuracy and expand the clinical testing field for a variety ages and gender.

\section{References}

[1] K. M. Meiburger, U. R. Acharya, and F. Molinari, "Automated localization and segmentation techniques for B-mode ultrasound images: A review," Comut. Biol. Med., vol. 91, no. 1, pp. 210-235, 2018.

[2] C. P. Loizou, C. S. Pattichis, and M. Pantziaris, T. Tyllis, A. Nicolaides, "Snakes based segmentation of the common carotid artery intima media," Med. Biol. Eng. Comput., vol. 45, no. 1, pp. 35-49, 2007.

[3] F. Valckx, and J. Thijssen, "Characterization of echographic image texture by cooccurrence matrix parameters," Ultrasound Med. Biol., vol. 23, no. 4, pp. 559-571, 1997.

[4] F. Molinari, C. Caresio, and U. Acharya, "Advances in quantitative muscle ultrasonography using texture analysis of ultrasound images," Ultrasound Med. Biol., vol. 41, no. 9, pp. 2520-253, 2015.

[5] B. Wiley, and B. Mohanty, "Handheld Ultrasound and Diagnosis of Cardiovascular Disease at the Bedside," J. Am. Coll. Cardiol., vol. 64, no. 2, pp. 229-230, 2014. 